\title{
Reply: Is CDIa involved in antitumour immune responses during carcinogenesis?
}

\author{
\begin{tabular}{l}
\hline BJ Coventry*, I and S Heinzel' \\
'Tumour Immunology Laboratory, Department of Surgery, University of Adelaide, Royal Adelaide Hospital, North Terrace, Adelaide 5000, South Australia
\end{tabular}
}

British Journal of Cancer (2004) 90, 939. doi: 10.1038/sj.bjc.660I598 www.bjcancer.com

(c) 2004 Cancer Research UK

Sir,

We agree with Dr Cappello's comment relating to our paper ${ }^{1}$ that the CD1a expression by dendritic cells (DC) in human cancers is a very interesting observation and that the possible role of the $\mathrm{CD} 1 \mathrm{a}$ molecule in the presentation of tumour-associated antigens requires further investigation. We were intrigued to note in the Cappello et al paper ${ }^{2}$ that $\mathrm{CD} 1 \mathrm{a}$ was expressed in $75-94 \%$ of epithelial biopsy tissue sections from patients with Barrett's metaplasia of the oesophagus, but that this was essentially not found in patients with dysplasia or neoplasia, nor in normal gastric or colonic tissues examined. Although the study was small, the report brief, and did not contain more advanced invasive carcinomas, it might indicate that transitory expression of $\mathrm{CD} 1 \mathrm{a}$ by epithelia may occur during the initial phases of carcinogenesis. We have some unsubstantiated data that also indicate that some in situ breast carcinomas may also show expression of CDla. It is unclear whether the expression of the CD1a molecules is actually on the epithelial cells per se or on intraepithelial DC. We have theorised that if CD1a expression occurs on both epithelial cells

\section{REFERENCES}

Cappello F, Rappa F, Bucchieri F, Zummo G (2003) CD1a as a novel biomarker for the diagnosis and the clinical outcome of Barrett's metaplasia. Lancet Oncol 4: 497 and DC, then this may indicate a similar arrangement to that observed for MHC molecular expression for presentation of peptide antigens. This would strengthen our hypothesis that CD1a, with some known structural similarity to MHC, may be very important for the presentation of tumour-derived glycolipid antigens to $\mathrm{T}$ cells. In addition, we are currently investigating $\mathrm{CD} 1 \mathrm{a}$ restricted $\mathrm{T}$-cell responses against tumour antigens, and we hypothesise that these are important for effective antitumour immune responses in vivo. If epithelial cells in transition towards neoplasia express CD1a together with neo-antigens, this may be a sufficient 'danger' signal to alert the immune system to early carcinogenesis, thus inducing an effective immune response in some individuals - perhaps as a mechanism for epithelial protection against further malignant progression. The failure of expression of CD1a by epithelia in transition may not allow an immune response to occur, perhaps explaining the worse prognosis of CDla-negative oesophageal lesions noted in the study of Cappello et al. This theory remains to be (dis)proved.
Coventry BJ, Morton J (2003) CD1a-positive infiltrating-dendritic cell density and 5-year survival from human breast cancer. $B r$ J Cancer 89: $533-538$

\footnotetext{
*Correspondence: BJ Coventry;

E-mail: brendon.coventry@adelaide.edu.au
} 